\title{
ARTICLE
}

\section{A comparative pharmacogenomic analysis of three classic TCM prescriptions for coronary heart disease based on molecular network modeling}

\author{
Ying-ying Zhang ${ }^{1,2}$, Zi-de Zhao ${ }^{3}$, Peng-yun Kong ${ }^{4}$, Lin Gao ${ }^{4}$, Ya-nan Yu ${ }^{2}$, Jun Liu ${ }^{2}$, Peng-qian Wang ${ }^{2,5}$, Bing Li $^{2}$, Xiao-xu Zhang $^{3}$, \\ Li-qiang Yang ${ }^{4}$ and Zhong Wang ${ }^{2}$
}

Traditional Chinese medicine (TCM) has evolved over several thousands of years, which has been shown to be efficacious in the treatment of ischemic heart disease. Three classical TCM prescriptions, namely Xuefu Zhuyu Decoction, Zhishi Xiebai Guizhi Decoction, and Gualou Xiebai Banxia Decoction, have been extensively used in the treatment of coronary heart disease (CHD). Based on molecular network modeling, we performed a comparative pharmacogenomic analysis to systematically determine the drug-targeting spectrum of the three prescriptions at molecular level. Wide-area target molecules of CHD were covered, which was a common feature of the three decoctions, demonstrating their therapeutic functions. Meanwhile, collective signaling involved metabolic/pro-metabolic pathways, driving and transferring pathways, neuropsychiatric pathways, and exocrine or endocrine pathways. These organized pharmacological disturbance was mainly focused on almost all stages of CHD intervention, such as anti-atherosclerosis, lipid metabolism, inflammation, vascular wall function, foam cells formation, platelets aggregation, thrombosis, arrhythmia, and ischemia-reperfusion injury. In addition, heterogeneity analysis of the global pharmacological molecular spectrum revealed that signaling crosstalk, cascade convergence, and key targets were tendentious among the three decoctions. After all, it is unadvisable to rank the findings on targeting advantages of the three decoctions. Comparative pharmacological evidence may provide an appropriate decoction scheme for individualized intervention of CHD.

Keywords: classical TCM prescriptions; coronary heart disease; Xuefu Zhuyu Decoction; Zhishi Xiebai Guizhi Decoction; Gualou Xiebai Banxia Decoction; molecular network modeling; network pharmacology

Acta Pharmacologica Sinica (2020) 41:735-744; https://doi.org/10.1038/s41401-019-0352-3

\section{INTRODUCTION}

Coronary heart disease (CHD) remains a major health burden worldwide, although the mortality rate has been declining over the past few decades $[1,2]$. Optimal pharmacological therapy for ischemic heart disease, includes an angiotensin converting enzyme (ACE) inhibitor, beta-blocker, statin, aspirin, etc. However, it is troubling that before giving prescriptions, clinicians need to carefully screen dozens of contraindications for patients such as pregnancy or lactation or liver or kidney dysfunction. Adverse reactions and toxicities have limited the clinical use of drugs. Recently, systems biology studies have shown that for complex diseases, Chinese medicine formulas have the advantages of multitarget interventions and minimal side effects $[3,4]$. In China, decoctions including Xuefu Zhuyu (XFZY), Zhishi Xiebai Guizhi (ZSXBGZ), and Gualou Xiebai Banxia (GLXBBX) have contributed to fighting against coronary artery disease for a long time. However, the critical issue must be "How to do?" and "What is it?" for distinguishing the three decoctions at the molecular level, which involves the "different treatments for the same disease" concept of TCM.

Network simulation of the multitarget action of drugs is a fascinating work because it may provide an opportunity to observe a particular angle of the pharmacological nature of a drug. The development of network pharmacology provides an effective solution for decoding drug combinations, such as triggering divergence/convergence pathways or concentrated/ integrated regulation $[3,5]$. Here, we use the known pharmacogenomic data and molecular network modeling to distinguish the target spectrum of the three formulas from easily confused pharmacodynamics and then determine the principle of formula screening for precise interventions for coronary heart disease.

\footnotetext{
${ }^{1}$ Dongzhimen Hospital, Beijing University of Chinese Medicine, Beijing 100700, China; ${ }^{2}$ Institute of Basic Research in Clinical Medicine, China Academy of Chinese Medical Sciences, Beijing 100700, China; ${ }^{3}$ Eye Hospital, China Academy of Chinese Medical Sciences, Beijing 100040, China; ${ }^{4}$ Guangxi University of Chinese Medicine, Nanning 530200, China and ${ }^{5}$ Institute of Chinese Materia Medica, China Academy of Chinese Medical Sciences, Beijing 100700, China

Correspondence: Li-qiang Yang (ylq6606@163.com) or Zhong Wang (zhonw@vip.sina.com)

These authors contributed equally: Ying-ying Zhang, Zi-de Zhao, Peng-yun Kong
}

Received: 23 July 2019 Accepted: 17 December 2019

Published online: 12 February 2020 


\section{MATERIALS AND METHODS}

Related molecules of XFZY decoction, ZSXBGZ decoction, and GLXBBX decoction

All drugs included in the XFZY decoction, ZSXBGZ decoction, and GLXBBX decoction were searched in the Compound Reference Database, Chinese Academy of Sciences (http://www.chemcpd.csdb. $\mathrm{cn} /$ ) and the National Scientific Data Sharing Platform for Population and Health (http://www.ncmi.cn/) to identify all of the known chemical compositions of each decoction. Then, these decoctionrelated compound data were entered into the STITCH database (http://stitch.embl.de/) [6]. Species were set to "Homo sapiens", and thus, the related molecular information could be obtained.

\section{CHD-related gene acquisition and network construction}

In the OMIM database (https://www.ncbi.nlm.nih.gov/omim/) search interface, "coronary heart disease" was entered to gain access to CHD-related genes. The related genes were uploaded to the Search Tool for Recurring Instances of Neighboring Genes (STRING) database version 9.05 (http://string905.embl.de/) to characterize the associations between these molecules and to generate molecular interaction networks using Homo sapiens as the background. The CHD-related network was obtained.

Comparison of the three decoctions in treating $\mathrm{CHD}$

The related molecules of the XFZY decoction, ZSXBGZ decoction, and GLXBBX decoction were mapped to the CHD-related network, and the similarities and differences of the three decoctions were compared.

Functional enrichment analysis

The functional enrichment analyses of the three decoctions were performed by the Database for Annotation, Visualization, and Integrated Discovery (DAVID; https://david-d.ncifcrf.gov/) version 6.7 (parameters, count $=2$, expression analysis systematic explorer $=0.01$, background and species $=$ Homo sapiens). The $P$ values of the Kyoto Encyclopedia of Genes and Genomes (KEGG) pathway were ranked. A Fisher exact $P$ value of 0 represents perfect enrichment. A $P<0.01$ was considered to indicate a statistically significant difference in the annotation categories. CHD-related signaling pathways were obtained from the comparative toxicogenomics database (CTD; http://ctdbase.org/).

The pathological mechanism of CHD

Based on the published literature (PubMed, https://www.ncbi.nlm. nih.gov/pubmed/), information about the pathological mechanism of CHD was extracted to outline the pathology of CHD.

\section{RESULTS}

Related compounds and molecules of the XFZY decoction, ZSXBGZ decoction, and GLXBBX decoction

Based on the Compound Reference Database, Chinese Academy of Sciences (http://www.chemcpd.csdb.cn/) and the National Scientific Data Sharing Platform for Population and Health (http://www.ncmi.cn/), for three formulas XFZY decoction (peach kernel, Bupleurum, Angelica, radix paeoniae rubrathe, Achyranthes, Radix Rehmanniae, Ligusticum wallichii, Platycodon grandiflorum, safflower, fructus aurantii), ZSXBGZ decoction (Fructus Aurantii Immaturus, Trichosanthes kirilowii Maxim, Allium macrostemon Bunge, Magnolia officinalis, Cassia twig), GLXBBX decoction (Allium macrostemon Bunge, Pinellia ternata, Trichosanthes kirilowii Maxim, liquor), we obtained 667 related compounds of XFZY decoction, 224 related compounds of ZSXBGZ decoction, and 169 related compounds of GLXBBX decoction (Supplementary Tables S1-S3). After the names of these compounds were searched in the STITCH database and only high quality (higher than 0.9 ) results were extracted, we obtained 2030 related molecules of XFZY decoction, 1322 related molecules of ZSXBGZ decoction, and 1600 related molecules of GLXBBX decoction (Supplementary Tables S4-S6). There were 1236 overlapping molecules among the three decoctions, 598 unique molecules in the XFZY decoction, 21 unique molecules in the ZSXBGZ decoction, and 213 unique molecules in the GLXBBX decoction (Fig. 1a).

Related genes and networks of CHD

Based on the OMIM database, we obtained 465 CHD-related genes (Supplementary Table S7). Among them, there were 1725 direct interactions between 167 genes (score $\geq 0.4$ ). When the three decoction-related molecules were compared with CHD-related molecules, 88 overlapping molecules were identified (Fig. 1a). When the 167 genes were compared with CHD-related molecules, $82(93.18 \%$ of 88$)$ overlapping molecules were noted (Fig. 1b), and the other 6 nonoverlapping molecules were GLA, LDLR, HMGCR, CDKN2A, TNF, and HGD. Therefore, we might focus on the network consisting of the 167 molecules and these direct interactions and then compare the relationship between the 3 decoctions and the network. Of the 82 overlapping molecules, 19 were markers or targets for CHD (Fig. 1C), and 2 of the 6 nonoverlapping molecules (LDLR and TNF) were markers or targets for CHD. Among the 42 overlapping molecules between the 3 decoctions and the network of CHD, 11 (NOS3, PLA2G7, APOA1, ANGPTL4, APOB, CD36, APOE, MTHFR, APOC3, APOA5, and GNB3) were markers of CHD.

When the molecules of the three decoctions were compared with those of CHD network, 42 overlapping molecules were found, including only 1 overlapping molecule between the XFZY decoction and the ZSXBGZ decoction and CHD, 10 between the XFZY decoction and the GLXBBX decoction and CHD, 24 between the XFZY decoction and CHD, and 5 between the GLXBBX decoction and CHD (Fig. 2).

The top hub nodes with higher degrees in the CHD network model were listed as candidate hubs, the details that the three decoction groups targeting hub proteins (APOE, NFKB1, ESR1, SERPINE1, IFNG, ACTA2, APOB, TTR, APOA1, CCL2, PON1) were listed in Fig. 3.

Functional enrichment analysis of the three decoctions After the related molecules were entered into the DIVID software based on a $P<0.01$ indicating a statistically significant singling pathway, a total of 97,94 , and 82 KEGG pathways were identified from the XFZY decoction, GLXBBX decoction, and ZSXBGZ decoction, respectively (Supplementary Table S8-S10). Based on the CTD database, we obtained 106 related KEGG pathways of CHD (Supplementary Table S11). Thirty-six KEGG pathways from the three decoctions were related to CHD, and 26 KEGG pathways overlapped with those of CHD (Fig. 4, Supplementary Table S12). Based on these findings, we could compare the similarities and differences in the pharmaceutical mechanisms of the three decoctions.

Taking the PPAR signaling pathway as an example, which was one of the CHD-related pathways, all three decoctions had an impact on this pathway (Fig. 5). Moreover, it could be seen that the three decoctions had both similar and different effects on this signaling pathway.

Diagram of the pathological mechanism of CHD

Based on the PubMed database, we extracted the triggers and pathological mechanism of CHD (Fig. 6), which might help us better understand the mechanism of drug intervention and mutual links among individual drugs.

\section{DISCUSSION}

Network modeling-based drug-targeting spectrum TCM decoctions are characterized by the inclusion of a large family of effective components. The multicomponent nature of 

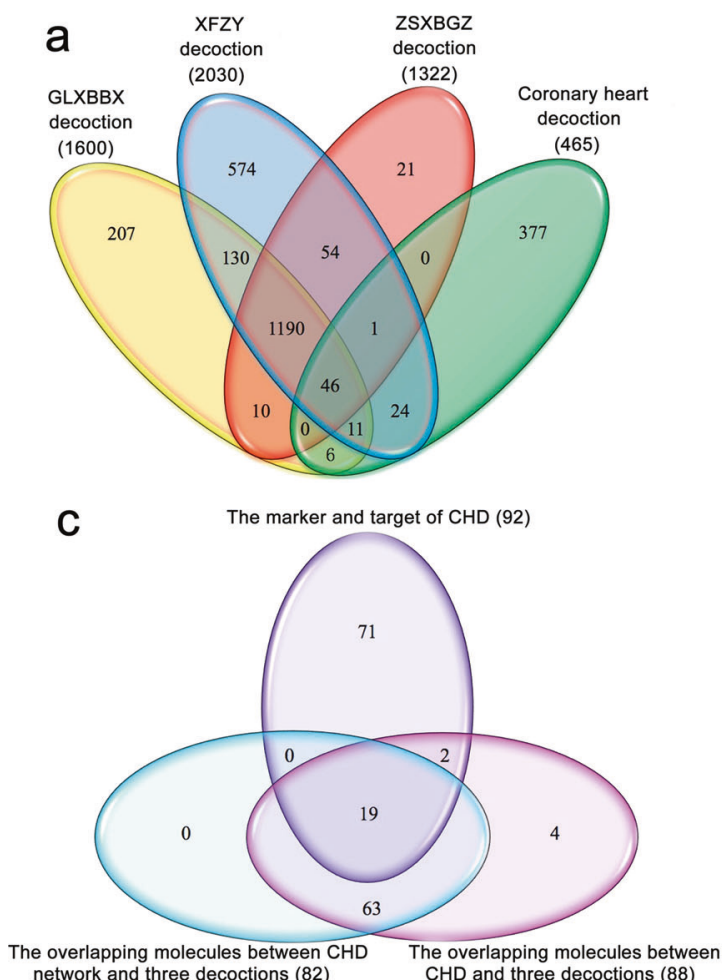

Fig. 1 The related molecules of the three decoctions and CHD. $\mathbf{a}$ The overlapping molecules among the three decoctions and CHD. $\mathbf{b}$ The overlapping molecules among the three decoctions and the network of CHD. c The markers and targets of CHD in comparison with the overlapping molecules between the CHD network (both in and out) and the three decoctions.

TCM decoctions can often confuse people because of the nontraceability of multitarget disturbances. Therefore, the concept of network pharmacology has been presented, emphasizing that the actions of multitarget drugs often surpass the actions of single-target drugs $[7,8]$. In this study, we analyzed hundreds of compounds and thousands of their well-founded acting molecules from each decoction. In addition, pathology-related molecules of CHD were analyzed for tracing the drug-targeting spectrum and deleting redundant disturbance information. Networks were constructed with 167 interactive molecules of CHD, and the validity of the network model was proved by Wayne analysis based on compound coverage of the marker-target of CHD (Fig. 1).

The drug targets of the XFZY, ZSXBGZ, and GLXBBX decoctions were mapped onto the CHD network (Fig. 2a-C), and details of the hit range were recognizable. The node color switch of the network model seemed to imply that the three decoction groups were biased with homogeneity and heterogeneity of the targeting spectrum. To improve the resolution of spectrum recognition, we fused the network with colorful nodes for labeling overlapping or isolated targets (Fig. 2d). Then, we came to a few interesting conclusions regarding the three decoctions: (1) the node hit rate was significant in the three decoction groups; (2) numerous shared targets were an essential feature; (3) the targeting spectrum was more similar between ZSXBGZ and GLXBBX; (4) the drug targets from ZSXBGZ were absolutely covered by those from XFZY; and (5) $X F Z Y$ and GLXBBX had their own unique drug targets.

Regardless of whether the target spectrum was similar or identical, the shared molecular background might drive directed pharmacological attacks to the marker-target network of CHD. This similarity was considered to be possible to construct a drug prediction model via the interaction between chemical space and genomic space [9]. Another work that we have discussed was a similar target spectrum of different types of diseases for developing copathology and shared drugs [10]. The specificity of the target spectrum is the motive driving drug development. This specificity is traceable in terms of network topology and functional annotations. Thus, the network molecular spectrum may be helpful for systematically discerning pharmacological characteristics due to the advantages of visualization, perspective, comparability, and flexibility.

Shared signaling pathways of pharmacogenomics

The response of biological cells to drugs depends more on signaling pathways. It is important to decide whether the molecule node in the network could be validated as a member of the pathway to eliminate "path orphans" [11]. Comfortingly, the KEGG pathway database has been constantly updating new perspectives on genomes, pathways, diseases, and drugs [12]. After the three groups of decoction network models were annotated with pathways, 22 shared and several unshared signaling pathways associated with CHD were labeled (Fig. 4). The evidence-based pathology cascade of CHD was mapped [13], and then efforts were devoted to find the response site of the pathway triggered by drugs (Fig. 5). In this case, the high-frequency shared signaling pathway might be involved in the routine mechanism of reversing the pathology of CHD (Supplementary Table S11), which could be integrated into several main categories, and part of the core signaling pathways are listed below.

Metabolic/pro-metabolic pathways. In terms of lipid metabolism, an adipocytokine signaling pathway participates in almost all pathological links of CHD [14] and can mediate the crosstalk between adipose tissues, the heart and the vasculature. Peroxisome proliferator-activated receptors (PPARs) can be widely expressed in adipose tissue, vascular smooth muscle tissue and myocardial tissue. The PPAR signaling pathway is also a regulator with the potential to reverse several key pathological mechanisms of CHD (Fig. 5), such as vascular wall function, foam cell formation, platelet aggregation, and thrombosis [15-18]. Fat digestion and absorption, 


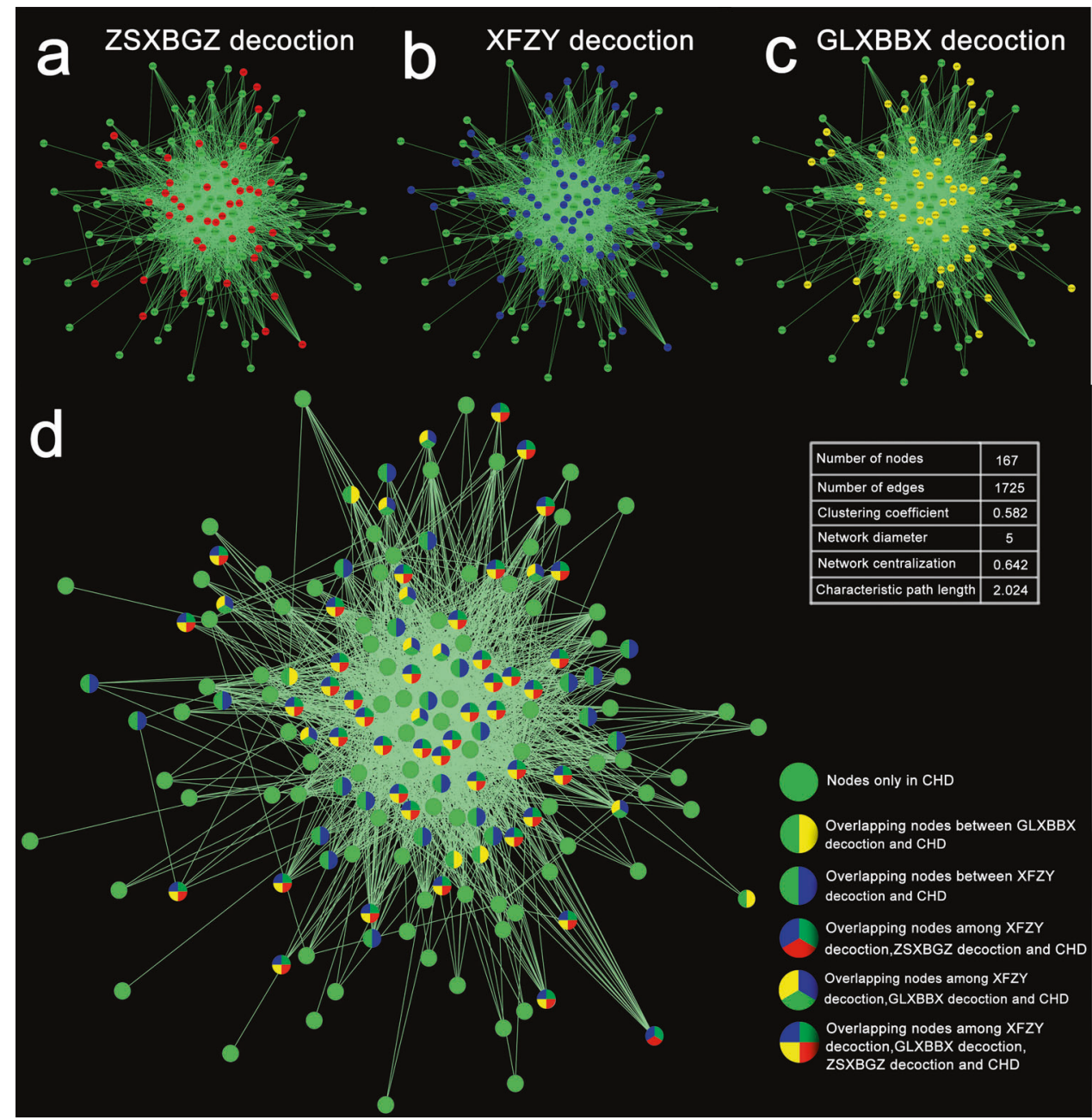

Fig. 2 The network of CHD intervened by the three decoctions. a-c Marked with red, blue, and yellow nodes indicate the molecules intervened by the ZSXBGZ, XFZY, and GLXBBX decoctions, respectively. d The overlapping and unique molecules in the CHD network intervened by the three decoctions.

arachidonic acid metabolism, and ether lipid metabolism are commonly implicated in CHD or other secondary-level etiology $[6,19,20]$, such as arteriosclerosis [21], the pro-inflammatory process [22], and vascular endothelial dysfunction [23].

Driving and transferring pathways. There are 19 members of the chemokine family involved in all stages of cardiovascular disease [24]. These cytokines may contribute to arteriosclerosis formation in the early stage, arrhythmia, or ischemia-reperfusion injury in the moderate-advanced stage, and dilated cardiomyopathy or heart failure in the late stage. The calcium signaling pathway is a hub induction switch in CHD that is involved in the inflammatory process of atherogenesis [25] and affects coronary endothelial function [26], foam cell formation [27], vascular tension adjustment [28], platelet aggregation [29], and vascular smooth muscle cell (VSMC) and fibroblast proliferation [30, 31]. The relationship between calcium signaling and arrhythmia has been deeply analyzed [32]; this association can be caused by calcium-channelrelated heritable mutations or acquired diseases of the myocardium. The gap junction allows intercellular communication in the cardiovascular system through connexons for the maintenance of the normal cardiac rhythm, endothelial function and the regulation of vascular tone as well as thrombosis control [33, 34]. Histological research has also shown that atheroma formation might be due to the loss of gap junction proteins, leading to increased permeability of the coronary artery [35].
Neuropsychiatric pathways. In this category, long-term depression is more prospectively associated with CHD [36]. Under the premise of confirmed relevance via high-level clinical evidence, two types of mechanisms are hypothesized [37]: one is the biological mechanism, including cardiac autonomic dysfunction, inflammatory reactions, endothelial dysfunction, and platelet dysfunction; the other is the behavioral mechanism, involving sedentary behavior and poor adherence to medication, diet, exercise, and smoking cessation.

Exocrine or endocrine pathways. Our understanding of the relationship between CHD and exocrine/endocrine function is still limited. Dietary fat digestion and absorption is initially exposed to oral saliva secretions, which may be the starting factor of abnormal lipid metabolism and obesity [38]. In addition, saliva components are increasingly deemed biomarkers that are used to evaluate the inflammation level and endothelial function of CHD patients $[39,40]$. Mass spectrometry analysis has further identified the salivary peptidome, a novel biomarker for the early diagnosis of cardiovascular disease patients with obstructive sleep apnea [41]. Bile acid is an important component of bile secretion, which reduces cardiovascular risk by lowering cholesterol levels [42]. Compared with patients without coronary artery disease (CAD), CAD patients have significantly decreased bile acid excretion levels, which may be a risk factor in the process of atherosclerosis [43]; in addition, bile acid may modulate endothelial function as 


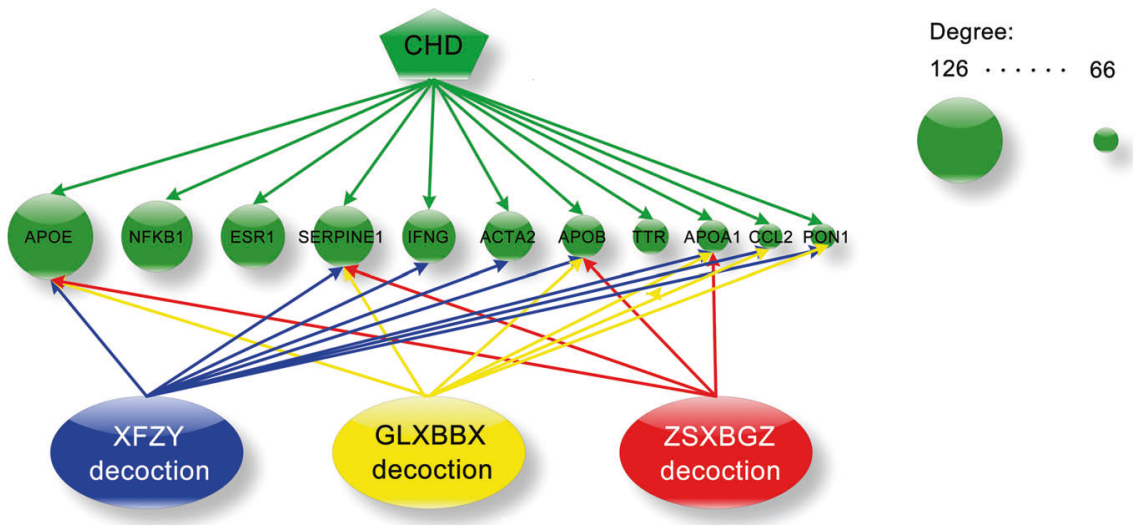

Fig. 3 The top hub in the CHD network intervened by the three decoctions. The green nodes denote the top 11 hubs in the CHD network, and the lines with red, blue, and yellow colors connecting the three decoctions indicate the hubs intervened by the ZSXBGZ, XFZY, and GLXBBX decoctions, respectively.

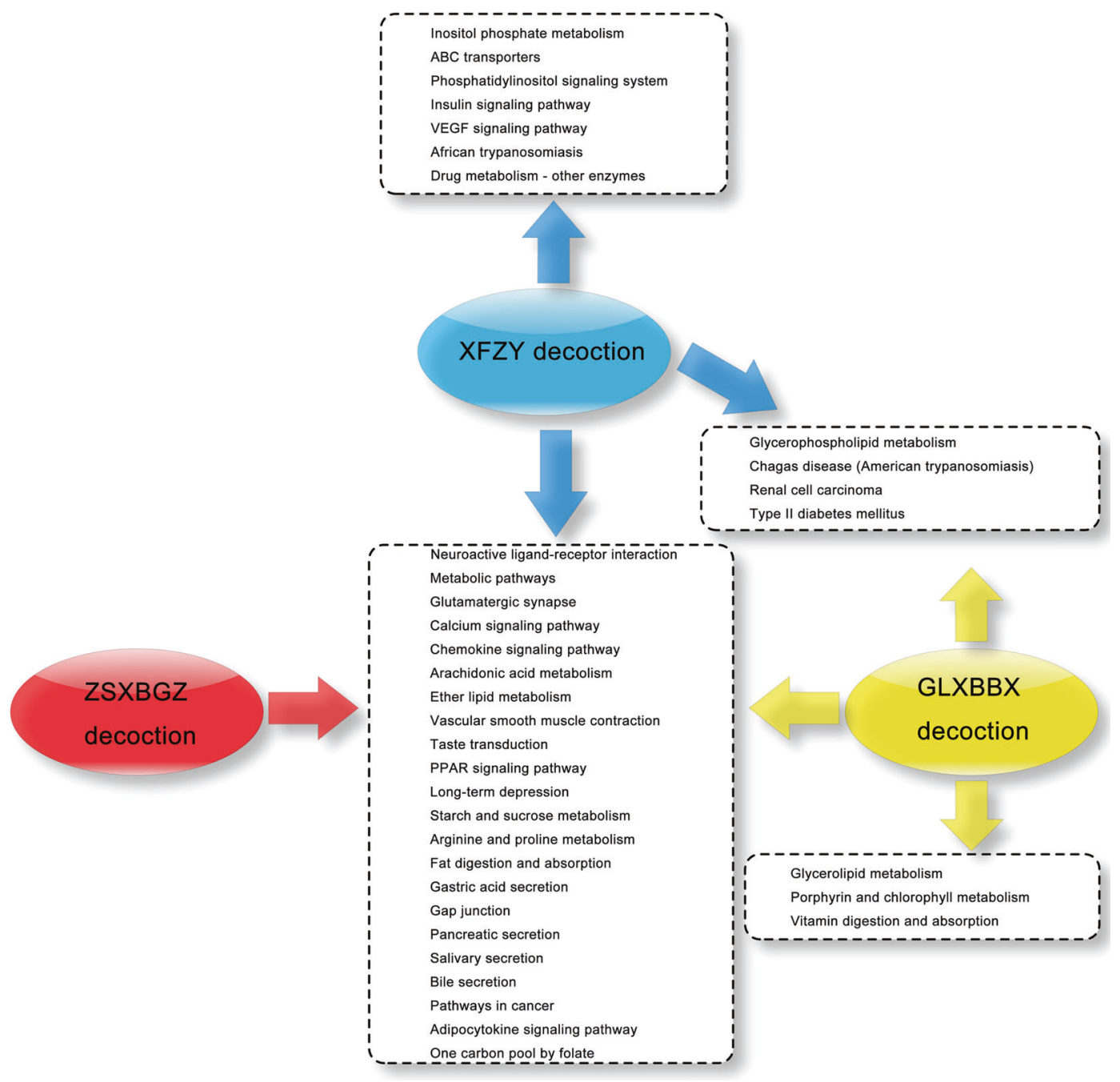

Fig. 4 The KEGG pathways between the three decoctions and CHD. The overlapping and unique KEGG signaling pathways between the three decoctions and CHD.

vascular endothelial cells express the $\mathrm{G}$ protein-coupled bile acid receptor [44].

Other shared core signaling pathways. Vascular smooth muscle contraction is the organizational behavior in response to modulation. The normal function of VSMCs is beneficial and protective for making atherosclerotic plaques stable [45]. This benefit may exist throughout the process, from the initial formation of foam cells to metalloproteinase-mediated plaque degradation. As a paradigm, shared pathogenic crosstalk also links cancer to CHD [46]. Common risk factors for both diseases have been explored to highlight potential biological mechanisms, such 


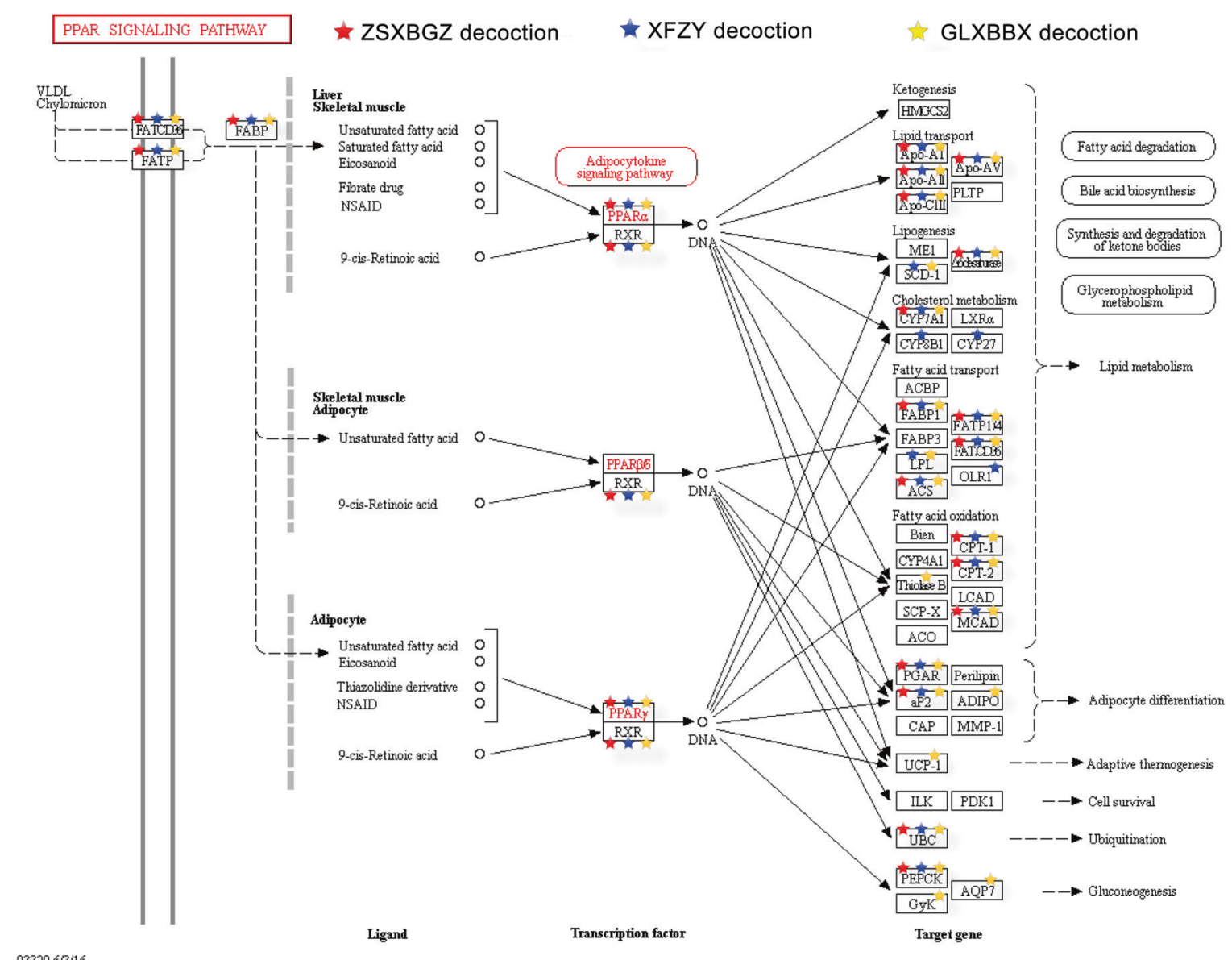

Fig. 5 PPAR signaling pathway. The PPAR signaling pathway is shown as an example of the overlapping pathways among the three decoctions. The red, blue and yellow stars represent the molecules affected by the ZSXBGZ, XFZY, and GLXBBX decoctions, respectively.

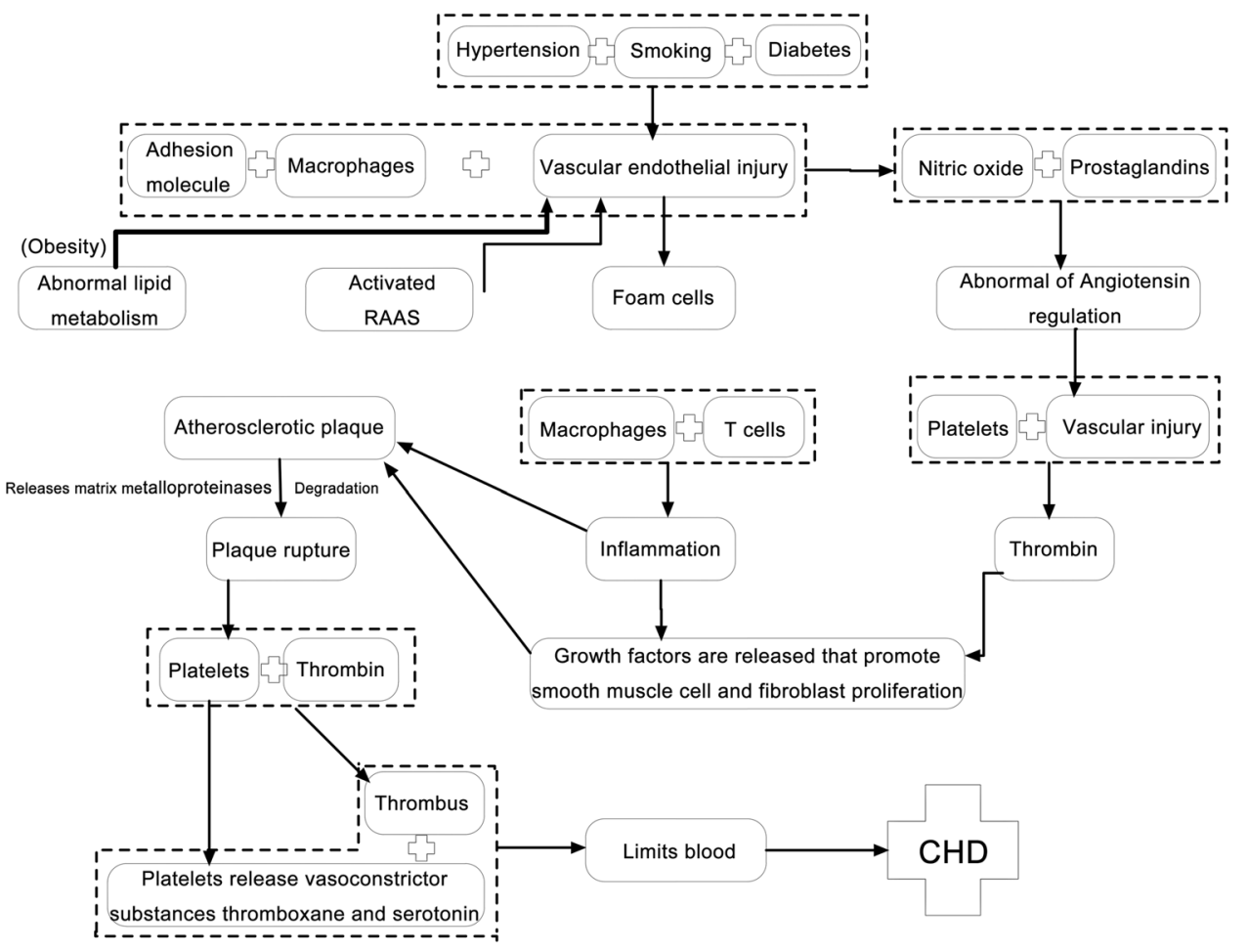

Fig. 6 Summary of the pathogenesis of CHD. 
as atherosclerosis, vascular endothelial function, vascular tension, smooth muscle cell function, inflammation, abnormal lipid metabolism, and obesity.

Comparable signaling pathways of pharmacogenomics Different from shared signaling pathways, comparability emphasizes the individualization of signaling navigation after drug use (Fig. 4), just like a drug signature that can help us to make a choice to fit a special indication.

1. Out of the several discriminating signaling pathways of the XFZY decoction molecular network, inositol phosphate metabolism and phosphatidylinositol signaling are important transmembrane signaling pathways that can release the second messenger inositol 1,4,5-trisphosphate (IP3). IP3/IP3 receptor signaling has been confirmed to be responsible for the transmission of apoptotic information following crosstalk with the gap junction pathway and calcium signaling pathway [47]. As a main regulator, the IP3R participates in $\mathrm{Ca}^{2+}$ release and cx43 phosphorylation, thereby affecting gap junction intercellular communication in cardiomyocytes [48]. Cardiovascular actions of insulin contribute to physiological hemodynamic homeostasis [49] because these phosphatidylinositol 3-kinase (PI3K)-dependent actions can stimulate the release of nitric oxide from the endothelium. Blocking the PI3K-dependent pathways can enhance the effects of insulin or vascular endothelial growth factor (VEGF) to increase the expression of those adhesion molecules, including intercellular adhesion molecule-1, vascular cell adhesion molecule (VCAM-1), and E-selectin [50]. These adhesion molecules may be extremely important for the regulation of circulating inflammation and vascular endothelial function. In the heart, insulin can dilate coronary arteries to improve myocardial perfusion and affect lipid metabolism [51, 52]. In addition, VSMC contractility would be decreased because of insulin regulating agonist-induced increases in cytosolic calcium through voltage-sensitive calcium channels [53], while it can also be increased when the sympathetic nervous system is involved [54]. The ATP-binding cassette (ABC) transporters form one of the largest known protein families, whose members $A B C A 1$ and $A B C G 1$ can act to transfer cholesterol to mature high-density lipoproteins (HDL) particles [55]. This feed-forward-like transfer process of mobilization and excretion of cholesterol and other lipids can prevent atherosclerosis, which is termed reverse cholesterol transport.

2. Three unique signaling pathways were enriched from the molecular network of GLXBBX decoction. Glycerolipid metabolism in genome-wide association studies of CHD has been analyzed [56], and its metabolizing enzymes are widely expressed in the ventricle and cardiac myocytes, most likely implicating ischemia. Lipolysis determines the process of inflammation during the development of atherosclerosis [57], even fatal obesity [58]. Glycerolipid metabolism can protect the heart from lipid accumulation under conditions that normalize PPAR-a expression [59]. Vitamin digestion and absorption is another pathway that is strongly involved in preventing cardiovascular disease. In particular, vitamin $\mathrm{E}$, a fat-soluble antioxidant vitamin, could be a key to preventing atherosclerosis [60], specifically, blocking the inflammatory response, foam cell formation, endothelial cytotoxicity, macrophage motility, and nitric oxide-induced vasodilatation. Randomized controlled trials showed that vitamin D supplementation is beneficial for the protection of heart failure [61]. Vitamin D receptors are found in almost all cardiovascular cell types, and vitamin D metabolites in pathways have been shown to regulate a number of pathways related to cardiovascular disease, including inflammation, thrombosis, and vasoconstriction [62]. The relationship between serum vitamin C levels and cardiovascular outcomes remains unknown. The improvements in lipid profiles, arterial stiffness, and endothelial function seem to support the use of vitamin $C$ supplements to reduce the risk and mortality of cardiovascular disease [63], and further evidence needs to be provided. Moreover, vitamin B (12) together with folic acid are involved in the pathogenesis of cardiovascular disease [64], mainly depending on higher levels of serum homocysteine.

3. One overlapping pathway between the molecular network of the XFZY decoction and the GLXBBX decoction is glycerophospholipid metabolism. In recent studies, this previously unreported pathway was shown to be associated with atherosclerosis based on the pathway analysis of plasma metabolites at the pathological stage [65]. Renal cell carcinoma is not a routine pathway for CHD pathology signaling clusters. It has been reported that local atherosclerosis is more distinct in tumor-positive than in tumornegative renal specimens [66]. Conversely, high-frequency correlations are shown between type 2 diabetes and CHD [67], such as high levels of postprandial plasma glucose, total cholesterol, low-density lipoproteins (LDLs), and triglycerides and low levels of HDLs. Furthermore, a wideranging prevention strategy is able to improve insulin sensitivity and reduce insulin resistance as well as cardiovascular risk factors [68].

It now appears that combined with these particular pathways, most pathways regulated by the XFZY decoction exhibit strong and frequent interpathway crosstalk, highlighting robust convergence of the signaling cascade. Pathway cooperation in nested or multilayer hierarchical forms can perform biofunctions more integrally [69]. For example, intercellular communication in the cardiovascular system through the gap junction pathway, which dominates multi-pathway combinations including the gap junction-calcium-glutamatergicinositol phosphate-phosphatidylinositol pathway, inflammation and vascular endothelial function and lipid metabolism regulated by the insulin signaling pathway, plays a leading role in a wide range of interpathway crosstalk involving the type 2 diabetes mellitus-calcium-phosphatidylinositol-VEGF-vascular smooth muscle contraction-adipocytokine pathway. Similar to the above crosstalk paradigm-like gap junction community that also responded in the GLXBBX decoction according to data enrichment, only some of the pathway components involved are reduced. However, the pathway still has one noteworthy trigger-vitamin digestion and absorption in the GLXBBX decoction, whose family member metabolism is significantly associated with many pathologies of CHD. In addition, compared with the other two decoctions, the GLXBBX decoction did not have any individualized pathway response.

Decoding hub nodes of the network model in the three decoctions

The power-law degree distribution is used to characterize conventional biomolecular networks, which indicates a few hubs holding together numerous small nodes [70]. Dynamic information coupling of critical hub radiation-like links makes these nodes occupy the top of the high-level biological behavior, such as being responsible for cognition in the brain function network or immune response and lipid homeostasis in the cardiovascular disease network [71, 72]. In this study, the top 11 nodes with higher degrees in the CHD network model were listed as candidate hubs (Fig. 3), and the details that the 3 decoction groups targeting hub proteins were clear. If the hub node can be in charge of key regulation, it is worth looking forward to the prospect of the three decoctions for the treatment of CHD. Similar to pathway analysis as a whole, quantitative superiority for targeting hubs in XFZY 
decoction is outstanding, including 8 of the 11 hubs, i.e., APOE, SERPINE1, IFNG, ACTA2, APOB, APOA1, CCL2, and PON1. Degree calculation results in the addition of a candidate hub node.

According to the three colors of arrows in Fig. 3, cotargeted hubs of the three decoctions (APOA1, APOB, APOE, and SERPINE1) are also exactly unique in the ZSXBGZ decoction. Here, apolipoprotein family members are very active target hubs in response. APOA $1, A P O B$, and $A P O E$ are the main component proteins of $H D L, L D L$, and very low-density lipoprotein, respectively. The protection of atherosclerosis by HDL has already gone from hypothesis to validation. The preclinical experiment showing that the overexpression of major HDL protein APOA1 matched the epidemiological data for confirming the hypothesis [73], thereby making HDL a novel therapeutic target for preventing atherosclerosis. The malfunction of tissue cholesterol efflux has been proven in patients with a loss-of-function mutation in APOA1 by experimental evidence [74]. Anti-inflammatory, antioxidant, antiapoptotic, and NO-promoting effects could make APOA1 have the ability to be atheroprotective [75]. As better predictors of cardiovascular events, $\mathrm{APOB}, \mathrm{APOA} 1$, and the APOB/APOA1 ratio have been reported to be able to improve cardiovascular risk prediction [76]. Because LDL particles can increase the risk of atherothrombotic events via being easily internalized into the subintimal space where they adhere to matrix proteoglycans [77], and each particle has only one APOB molecule, the total APOB value indicates the total number of potentially atherogenic lipoproteins. Compared with other risk predictors, high APOB levels and a high APOB/APOA1 ratio showed stronger predictive performance in multivariate analyses. APOE, as a hub with the top degrees in this study, was initially described as a major ligand for LDL receptors with a role in cholesterol metabolism and the progression of atherosclerosis and cardiovascular disease. Analysis shows that the anti-atherosclerosis effect of APOE is mainly dependent on the clearance of circulating cholesterol [78]. In parallel, as subtypes of APOE, APOE2 increases atherogenic lipoprotein levels and APOE4 increases LDL levels, both enhancing the risk of heart disease together [78]. However, the association between APOE gene polymorphism and CHD is still not completely clear, which may require evidence from a large sample of epidemiological studies. SERPINE1 is a major physiological inhibitor of endogenous plasminogen activator, which can inhibit fibrin degradation, promote fibrin deposition in the vessel wall and stimulate smooth muscle cell proliferation. There is sufficient evidence to confirm the causal relationship between high plasma SERPINE1 concentrations and various thrombotic disorders $[79,80]$. Endothelial cell synthesis with platelet surface release increases the circulating concentration of SERPINE1. When this incremental mechanism is inhibited, the thrombolytic outcome may be altered [81]. A recent systematic review has indicated the causal effect of elevated SERPINE1 levels on CHD risk involving glucose dysfunction [82].

As one of the two shared target hubs between the XFZY decoction and GLXBBX decoction, CCL2, also called monocyte chemoattractant protein 1 (MCP-1), is considered to be involved in the pathogenesis of human atherosclerosis and myocardial infarction. Atherosclerotic plaque formation can be attributed to the chemotactic activity of MCP-1 to promote foam cell development [83]. A myocardial infarction cohort study further revealed that in this context, the significant clinical relevance of MCP-1 levels were age, cigarette smoking, triglycerides, body mass index, and waist-to-hip ratio [84]. CCL2 also has a powerful modulation of fibroblasts and endothelial cell phenotypes and thus may promote myocardial infarction healing [85]; otherwise, CCL2 deficit may lead to weakened tissue remodeling postinfarction at the expense of a prolonged inflammatory phase. PON1 is another shared hub node between the two decoctions and is a calcium-dependent HDL-associated lactonase. Studies have shown that PON1 can both significantly reduce lipid peroxide generation and provide HDL-associated protection against atherosclerosis [86]. These anti-atherosclerosis processes mainly depend on reducing macrophage oxidation, foam cell formation and homocysteine levels as well as promoting HDL cholesterol efflux. Interestingly, PON1 and MCP-1 seem to be coincidentally packaged into a pair of hubs with up-/downstream regulatory relationships. It has been reported that oxidized fatty acids have anti-atherogenic effects only after being hydrated by PON1. Otherwise, unhydrated oxidized fatty acids with strong pro-inflammatory properties can mediate MCP-1 production and attract monocytes into the endarterium [87]. Therefore, the expression of PON1 and MCP-1 regulated by the two decoctions may be an important way to fight against the early stage of CHD pathology.

IFNG and ACTA2 are the exclusive hubs of XFZY decoction. The gene IFNG encodes a soluble cytokine that is a member of the type II interferon class. Studies have shown that increased IFNG during infection can regulate the production of matrix metalloproteinases and their inhibitors, which are important in plaque stability [88]. Another clinical study revealed that serum IFNG levels were significantly positively correlated with TG concentration in CHD patients, which could be involved in the development of atherosclerosis of the coronary artery [89]. ACTA2, the human aortic smooth muscle actin (SMA) gene typically expressed in VSMCs, contributes to vascular motility and contraction and may cause a variety of vascular diseases in the event of mutation, such as coronary artery disease and multisystemic smooth muscle dysfunction syndrome [90, 91]. In experimental homozygous aSMA knockout mice, decreased vascular contractility and reduced basal blood pressure were notable, suggesting the important role of a-SMA in maintaining vascular tone [92]. The historical view of VSMCs in atherosclerotic plaques presents a double-edge sword. Both the harm and the benefit of VSMCs depend on the stage of plaque development [93]. Hence, drug-affected ACTA2 expression is crucial in the prevention and treatment of atherosclerosis.

\section{CONCLUSION}

This is a pioneering study based on a global molecular simulation between pharmacology and pathology to identify how three decoctions directionally fight against CHD. Shared signaling pathways and hub nodes as carriers of basic biological functions make us aware of why these prescriptions have long been active in first-line clinical practice in TCM. Dense and organized pharmacological disturbances mainly focused on almost all stages of CHD intervention, such as the prevention of atherosclerosis, lipid metabolism, inflammation, vascular wall function, foam cell formation, platelet aggregation, thrombosis, arrhythmia, and ischemia-reperfusion injury. In addition to these aforementioned shared presumptive pharmacological characteristics, highfrequency crosstalk, and strong convergence are the macroscopic features of the signaling pathway triggered by the XFZY decoction. In particular, inositol phosphate metabolism and phosphatidylinositol signaling are considered to be intermediate modulators of multiple signaling pathway linkages, which can be associated with intercellular communication in cardiomyocytes, circulating inflammation, and vascular endothelial function. In addition, for the XFZY decoction, reversing cholesterol transport by $A B C$ transporters, plaque stability, and vascular activity improvement are still independent potential preventive targets of cardiovascular circulatory disorders. The GLXBBX decoction is concentrated in the regulation of glyceride metabolism and the digestion and absorption of vitamins individually. In particular, multiple vitamins may respond to resistance to pathological processes such as inflammation, foam cell formation, endothelial cell toxicity, and thrombosis. In this study, the GLXBBX decoction did not have any independent targeting features; however, a larger scale of molecular mining and analysis is still needed. 
In summary, the method of comparative pharmacogenomic analysis provides us with an opportunity to gain insights into the large-scale molecular target differential spectrum characteristics of the three decoctions. By using the molecular network model, drug target tracing is clear, and then molecular data can be further discussed in an integrated manner at different dimensions comparatively. The high-frequency and similar target spectral features among the three decoctions in treating CHD were highlighted. Although it is unadvisable to rank the findings on targeting the advantages of the three decoctions, XFZY seems to have better pharmacological heterogeneity both in signaling pathways and in hub nodes. Comparative pharmacological evidence may provide an appropriate decoction scheme for individualized interventions for CHD.

\section{ACKNOWLEDGEMENTS}

This work was supported by the National Major Scientific and Technological Special Project for "Significant New Drugs Development" (No. 2017ZX09304019) and the National Natural Science Foundation of China (81603486).

\section{AUTHOR CONTRIBUTIONS}

YYZ and ZDZ wrote the paper and drew the figures; PYK sorted the data and created the tables; $L G, X X Z, P Q W$, and BL modified the tables and revised the paper; YNY and $J \mathrm{~L}$ revised the paper; and $\mathrm{LQY}$ and $\mathrm{ZW}$ directed the research and revised the paper.

\section{ADDITIONAL INFORMATION}

The online version of this article (https://doi.org/10.1038/s41401-019-0352-3) contains supplementary material, which is available to authorized users.

Competing interests: The authors declare no competing interests.

\section{REFERENCES}

1. Go AS, Mozaffarian D, Roger VL, Benjamin EJ, Berry JD, Blaha MJ, et al. Heart disease and stroke statistics-2014 update: a report from the American Heart Association. Circulation. 2014;129:e28-292.

2. Townsend N, Wilson L, Bhatnagar P, Wickramasinghe $K$, Rayner M, Nichols $M$. Cardiovascular disease in Europe: epidemiological update 2016. Eur Heart J. 2016;37:3232-45

3. Wang Z, Liu J, Cheng Y, Wang Y. Fangjiomics: in search of effective and safe combination therapies. J Clin Pharmacol. 2011;51:1132-51.

4. Duan DD, Wang Z, Zhang BL, Wang YY. Fangjiomics: revealing adaptive omics pharmacological mechanisms of the myriad combination therapies to achieve personalized medicine. Acta Pharmacol Sin. 2015;36:651-3.

5. Zhang YY, Li HX, Chen YY, Fang H, Yu YN, Liu J, et al. Convergent and divergent pathways decoding hierarchical additive mechanisms in treating cerebral ischemia-reperfusion injury. CNS Neurosci Ther. 2014;20:253-63.

6. Graessler J, Schwudke D, Schwarz PE, Herzog R, Shevchenko A, Bornstein SR. Topdown lipidomics reveals ether lipid deficiency in blood plasma of hypertensive patients. PLoS ONE. 2009;4:e6261.

7. Poornima P, Kumar JD, Zhao Q, Blunder M, Efferth T. Network pharmacology of cancer: from understanding of complex interactomes to the design of multitarget specific therapeutics from nature. Pharmacol Res. 2016;111:290-302.

8. Csermely $\mathrm{P}$, Agoston $\mathrm{V}$, Pongor $\mathrm{S}$. The efficiency of multi-target drugs: the network approach might help drug design. Trends Pharmacol Sci. 2005;26:178-82.

9. Ding H, Takigawa I, Mamitsuka H, Zhu S. Similarity-based machine learning methods for predicting drug-target interactions: a brief review. Brief Bioinform. 2014:15:734-47.

10. Zhang Y, Kong P, Chen Y, Yu Y, Liu J, Yang L, et al. Significant overlapping modules and biological processes between stroke and coronary heart disease. CNS Neurol Disord Drug Targets. 2014;13:652-60.

11. Rahmati S, Abovsky M, Pastrello C, Jurisica I. pathDIP: an annotated resource for known and predicted human gene-pathway associations and pathway enrichment analysis. Nucleic Acids Res. 2017;45(D1):D419-26.

12. Kanehisa M, Furumichi M, Tanabe M, Sato $Y$, Morishima K. KEGG: new perspectives on genomes, pathways, diseases and drugs. Nucleic Acids Res. 2017;45(D1): D353-61.

13. Bagdasarian N. Andreoli and carpenter's cecil essentials of medicine. Jama. 2010;304:1615. https://doi.org/10.1001/jama.2010.1468.

14. Van de Voorde J, Pauwels B, Boydens C, Decaluwe K. Adipocytokines in relation to cardiovascular disease. Metabolism. 2013;62:1513-21.
15. Werner CM, Schirmer SH, Gensch C, Pavlickova V, Poss J, Wright MB, et al. The dual PPARalpha/gamma agonist aleglitazar increases the number and function of endothelial progenitor cells: implications for vascular function and atherogenesis. Br J Pharmacol. 2014:171:2685-703.

16. Chinetti G, Lestavel S, Bocher V, Remaley AT, Neve B, Torra IP, et al. PPAR-alpha and PPAR-gamma activators induce cholesterol removal from human macrophage foam cells through stimulation of the ABCA1 pathway. Nat Med. 2001;7:53-8.

17. Moraes LA, Spyridon M, Kaiser WJ, Jones Cl, Sage T, Atherton RE, et al. Nongenomic effects of PPARgamma ligands: inhibition of GPVI-stimulated platelet activation. J Thromb Haemost. 2010;8:577-87.

18. Jin $\mathrm{H}$, Gebska MA, Blokhin IO, Wilson KM, Ketsawatsomkron $\mathrm{P}$, Chauhan $A K$, et al. Endothelial PPAR-gamma protects against vascular thrombosis by downregulating P-selectin expression. Arterioscler Thromb Vasc Biol. 2015;35:838-44.

19. Cao H. Adipocytokines in obesity and metabolic disease. J Endocrinol. 2014;220: T47-59.

20. Nielsen MS, Schmidt EB, Stegger J, Gorst-Rasmussen A, Tjonneland A, Overvad K. Adipose tissue arachidonic acid content is associated with the risk of myocardial infarction: a Danish case-cohort study. Atherosclerosis. 2013;227:386-90.

21. Liberale L, Bonaventura A, Vecchie A, Matteo C, Dallegri F, Montecucco F, et al. The role of adipocytokines in coronary atherosclerosis. Curr Atheroscler Rep. 2017;19:10.

22. Fuster JJ, Ouchi N, Gokce N, Walsh K. Obesity-induced changes in adipose tissue microenvironment and their impact on cardiovascular disease. Circ Res. 2016;118:1786-807.

23. Virdis A. Endothelial dysfunction in obesity: role of inflammation. High Blood Press Cardiovasc Prev. 2016;23:83-5.

24. Dusi V, Ghidoni A, Ravera A, De Ferrari GM, Calvillo L. Chemokines and heart disease: a network connecting cardiovascular biology to immune and autonomic nervous systems. Mediators Inflamm. 2016;2016:5902947.

25. Yuan Z, Miyoshi T, Bao Y, Sheehan JP, Matsumoto AH, Shi W. Microarray analysis of gene expression in mouse aorta reveals role of the calcium signaling pathway in control of atherosclerosis susceptibility. Am J Physiol Heart Circ Physiol. 2009;296:H1336-43.

26. Climent B, Moreno L, Martinez P, Contreras C, Sanchez A, Perez-Vizcaino F, et al Upregulation of SK3 and IK1 channels contributes to the enhanced endothelial calcium signaling and the preserved coronary relaxation in obese Zucker rats. PLoS ONE. 2014;9:e109432.

27. Liang SJ, Zeng DY, Mai XY, Shang JY, Wu QQ, Yuan JN, et al. Inhibition of Orai1 store-operated calcium channel prevents foam cell formation and atherosclerosis. Arterioscler Thromb Vasc Biol. 2016;36:618-28.

28. Sato K, Yamazaki J, Nagao T. Modulatory role of endothelial calcium level in vascular tension of canine depolarized coronary arteries. Am J Physiol. 1998;274(2 Pt 2):H494-9.

29. Harper MT, Poole AW. Store-operated calcium entry and non-capacitative calcium entry have distinct roles in thrombin-induced calcium signalling in human platelets. Cell Calcium. 2011;50:351-8.

30. Bi D, Toyama K, Lemaitre V, Takai J, Fan F, Jenkins DP, et al. The intermediate conductance calcium-activated potassium channel KCa3.1 regulates vascular smooth muscle cell proliferation via controlling calcium-dependent signaling. J Biol Chem. 2013;288:15843-53.

31. Zhang X, Zhang T, Wu J, Yu X, Zheng D, Yang F, et al. Calcium sensing receptor promotes cardiac fibroblast proliferation and extracellular matrix secretion. Cell Physiol Biochem. 2014;33:557-68.

32. Landstrom AP, Dobrev D, Wehrens XHT. Calcium signaling and cardiac arrhythmias. Circ Res. 2017;120:1969-93.

33. Michela $P$, Velia V, Aldo $P$, Ada P. Role of connexin 43 in cardiovascular diseases. Eur J Pharmacol. 2015;768:71-6.

34. Vaiyapuri S, Flora GD, Gibbins JM. Gap junctions and connexin hemichannels in the regulation of haemostasis and thrombosis. Biochem Soc Trans. 2015;43:489-94.

35. Sun B, Chen Z, Gu J, Tse G, Jiang J, Huang F, et al. Tight junction proteins and gap junction proteins play important roles in high fat dietary atherosclerosis pathogenesis. Int J Clin Exp Pathol. 2016;9:7969-76.

36. Liu RT, Hernandez EM, Trout ZM, Kleiman EM, Bozzay ML. Depression, social support, and long-term risk for coronary heart disease in a 13-year longitudinal epidemiological study. Psychiatry Res. 2017;251:36-40.

37. Carney RM, Freedland KE. Depression and coronary heart disease. Nat Rev Cardiol. 2017;14:145-55.

38. Vors C, Drai J, Gabert L, Pineau G, Laville M, Vidal H, et al. Salivary composition in obese vs normal-weight subjects: towards a role in postprandial lipid metabolism? Int J Obes. 2015;39:1425-8.

39. Janket SJ, Meurman JH, Nuutinen $P$, Qvarnstrom $M$, Nunn $M E$, Baird $A E$, et al. Salivary lysozyme and prevalent coronary heart disease: possible effects of oral health on endothelial dysfunction. Arterioscler Thromb Vasc Biol. 2006;26:433-4. 
40. Out D, Hall RJ, Granger DA, Page GG, Woods SJ. Assessing salivary C-reactive protein: longitudinal associations with systemic inflammation and cardiovascular disease risk in women exposed to intimate partner violence. Brain Behav Immun. 2012;26:543-51.

41. Zheng H, Li R, Zhang J, Zhou S, Ma Q, Zhou Y, et al. Salivary biomarkers indicate obstructive sleep apnea patients with cardiovascular diseases. Sci Rep. 2014;4:7046.

42. Ross S, D'Mello M, Anand SS, Eikelboom J, Consortium CAD, Stewart AF, et al. Effect of bile acid sequestrants on the risk of cardiovascular events: a mendelian randomization analysis. Circ Cardiovasc Genet. 2015;8:618-27.

43. Charach G, Grosskopf I, Rabinovich A, Shochat M, Weintraub M, Rabinovich P. The association of bile acid excretion and atherosclerotic coronary artery disease. Ther Adv Gastroenterol. 2011:4:95-101.

44. Kida T, Omori K, Hori M, Ozaki H, Murata T. Stimulation of G protein-coupled bile acid receptor enhances vascular endothelial barrier function via activation of protein kinase A and Rac1. J Pharmacol Exp Ther. 2014;348:125-30.

45. Johnson JL. Emerging regulators of vascular smooth muscle cell function in the development and progression of atherosclerosis. Cardiovasc Res. 2014;103:452-60.

46. Koene RJ, Prizment AE, Blaes A, Konety SH. Shared risk factors in cardiovascular disease and cancer. Circulation. 2016;133:1104-14.

47. Decrock E, Krysko DV, Vinken M, Kaczmarek A, Crispino G, Bol M, et al. Transfer of $I P(3)$ through gap junctions is critical, but not sufficient, for the spread of apoptosis. Cell Death Differ. 2012;19:947-57.

48. Kang M, Lin N, Li C, Meng Q, Zheng Y, Yan X, et al. Cx43 phosphorylation on S279/ 282 and intercellular communication are regulated by IP3/IP3 receptor signaling. Cell Commun Signal. 2014;12:58.

49. Muniyappa R, Montagnani M, Koh KK, Quon MJ. Cardiovascular actions of insulin. Endocr Rev. 2007;28:463-91.

50. Montagnani M, Golovchenko I, Kim I, Koh GY, Goalstone ML, Mundhekar AN, et al. Inhibition of phosphatidylinositol 3-kinase enhances mitogenic actions of insulin in endothelial cells. J Biol Chem. 2002;277:1794-9.

51. Sundell J, Knuuti J. Insulin and myocardial blood flow. Cardiovasc Res. 2003;57:312-9.

52. Ouwens DM, Boer C, Fodor M, de Galan P, Heine RJ, Maassen JA, et al. Cardiac dysfunction induced by high-fat diet is associated with altered myocardial insulin signalling in rats. Diabetologia. 2005;48:1229-37.

53. Saito $F$, Hori MT, Fittingoff $M$, Hino $T$, Tuck ML. Insulin attenuates agonistmediated calcium mobilization in cultured rat vascular smooth muscle cells. J Clin Invest. 1993;92:1161-7.

54. Scherrer U, Sartori C. Insulin as a vascular and sympathoexcitatory hormone: implications for blood pressure regulation, insulin sensitivity, and cardiovascular morbidity. Circulation. 1997;96:4104-13.

55. Fitzgerald ML, Mujawar Z, Tamehiro N. ABC transporters, atherosclerosis and inflammation. Atherosclerosis. 2010;211:361-70.

56. de las Fuentes L, Yang W, Davila-Roman VG, Gu C. Pathway-based genome-wide association analysis of coronary heart disease identifies biologically important gene sets. Eur J Hum Genet. 2012;20:1168-73.

57. Hasham SN, Pillarisetti S. Vascular lipases, inflammation and atherosclerosis. Clin Chim Acta. 2006;372:179-83.

58. Prentki M, Madiraju SR. Glycerolipid metabolism and signaling in health and disease. Endocr Rev. 2008:29:647-76.

59. Rame JE, Barouch LA, Sack MN, Lynn EG, Abu-Asab M, Tsokos M, et al. Caloric restriction in leptin deficiency does not correct myocardial steatosis: failure to normalize PPAR\{alpha\}/PGC1\{alpha\} and thermogenic glycerolipid/fatty acid cycling. Physiol Genomics. 2011;43:726-38.

60. Saremi A, Arora R. Vitamin E and cardiovascular disease. Am J Ther. 2010;17: e56-65.

61. Ford JA, MacLennan GS, Avenell A, Bolland M, Grey A, Witham M, et al. Cardiovascular disease and vitamin $D$ supplementation: trial analysis, systematic review, and meta-analysis. Am J Clin Nutr. 2014;100:746-55.

62. Norman PE, Powell JT. Vitamin D and cardiovascular disease. Circ Res. 2014;114:379-93.

63. Moser MA, Chun OK. Vitamin C and heart health: a review based on findings from epidemiologic studies. Int J Mol Sci. 2016;17:1328.

64. Genser D, Prachar H, Hauer R, Halbmayer WM, Mlczoch J, Elmadfa I. Homocysteine, folate and vitamin b12 in patients with coronary heart disease. Ann Nutr Metab. 2006;50:413-9.

65. Dang VT, Huang A, Zhong LH, Shi Y, Werstuck GH. Comprehensive plasma metabolomic analyses of atherosclerotic progression reveal alterations in glycerophospholipid and sphingolipid metabolism in apolipoprotein E-deficient mice. Sci Rep. 2016;6:35037.

66. Hager M, Mikuz G, Kolbitsch C, Moser PL. Association between local atherosclerosis and renal cell carcinomas. Nutr Cancer. 2008;60:364-7.

67. Rani HS, Madhavi G, Rao VR, Sahay BK, Jyothy A. Risk factors for coronary heart disease in type II diabetes mellitus. Indian J Clin Biochem. 2005;20:75-80.
68. Haffner SM, Miettinen $\mathrm{H}$. Insulin resistance implications for type II diabetes mellitus and coronary heart disease. Am J Med. 1997;103:152-62.

69. Zhang Y, Wang Z, Wang Y. Multi-hierarchical profiling: an emerging and quantitative approach to characterizing diverse biological networks. Brief Bioinform. 2017;18:57-68.

70. Barabasi AL, Oltvai ZN. Network biology: understanding the cell's functional organization. Nat Rev Genet. 2004;5:101-13.

71. Achard S, Salvador R, Whitcher B, Suckling J, Bullmore E. A resilient, low-frequency, small-world human brain functional network with highly connected association cortical hubs. J Neurosci. 2006;26:63-72.

72. Diez D, Wheelock AM, Goto S, Haeggstrom JZ, Paulsson-Berne G, Hansson GK, et al. The use of network analyses for elucidating mechanisms in cardiovascular disease. Mol Biosyst. 2010;6:289-304.

73. Rader DJ, Hovingh GK. HDL and cardiovascular disease. Lancet. 2014;384:618-25.

74. Holleboom AG, Jakulj L, Franssen R, Decaris J, Vergeer M, Koetsveld J, et al. In vivo tissue cholesterol efflux is reduced in carriers of a mutation in APOA1. J Lipid Res. 2013;54:1964-71.

75. Luscher TF, Landmesser U, von Eckardstein A, Fogelman AM. High-density lipoprotein: vascular protective effects, dysfunction, and potential as therapeutic target. Circ Res. 2014;114:171-82.

76. Walldius G, Jungner I. Apolipoprotein B and apolipoprotein A-l: risk indicators of coronary heart disease and targets for lipid-modifying therapy. J Intern Med. 2004;255:188-205.

77. Hurt-Camejo E, Camejo G, Rosengren B, Lopez F, Wiklund O, Bondjers G. Differential uptake of proteoglycan-selected subfractions of low density lipoprotein by human macrophages. J Lipid Res. 1990:31:1387-98.

78. Yousuf FA, lqbal MP. Review: apolipoprotein E (Apo E) gene polymorphism and coronary heart disease in Asian populations. Pak J Pharm Sci. 2015;28:1439-44.

79. Thogersen AM, Jansson JH, Boman K, Nilsson TK, Weinehall L, Huhtasaari F, et al. High plasminogen activator inhibitor and tissue plasminogen activator levels in plasma precede a first acute myocardial infarction in both men and women: evidence for the fibrinolytic system as an independent primary risk factor. Circulation. 1998;98:2241-7.

80. Margaglione M, Di Minno G, Grandone E, Vecchione G, Celentano E, Cappucci G, et al. Abnormally high circulation levels of tissue plasminogen activator and plasminogen activator inhibitor-1 in patients with a history of ischemic stroke. Arterioscler Thromb. 1994;14:1741-5.

81. Kohler HP, Grant PJ. Plasminogen-activator inhibitor type 1 and coronary artery disease. N. Engl J Med. 2000;342:1792-801.

82. Song C, Burgess S, Eicher JD, O'Donnell CJ, Johnson AD. Causal effect of plasminogen activator inhibitor type 1 on coronary heart disease. J Am Heart Assoc. 2017;6: pii: e004918. https://doi.org/10.1161/JAHA.116.004918.

83. Niu J, Kolattukudy PE. Role of MCP-1 in cardiovascular disease: molecular mechanisms and clinical implications. Clin Sci (Lond). 2009;117:95-109.

84. McDermott DH, Yang Q, Kathiresan S, Cupples LA, Massaro JM, Keaney JF, et al. CCL2 polymorphisms are associated with serum monocyte chemoattractant protein-1 levels and myocardial infarction in the Framingham Heart Study. Circulation. 2005;112:1113-20.

85. Dewald O, Zymek P, Winkelmann K, Koerting A, Ren G, Abou-Khamis T, et al. $\mathrm{CCL} 2 /$ monocyte chemoattractant protein-1 regulates inflammatory responses critical to healing myocardial infarcts. Circ Res. 2005;96:881-9.

86. Mackness MI, Mackness B, Durrington PN, Fogelman AM, Berliner J, Lusis AJ, et al. Paraoxonase and coronary heart disease. Curr Opin Lipidol. 1998;9:319-24.

87. Mackness B, Hine D, Liu Y, Mastorikou M, Mackness M. Paraoxonase- 1 inhibits oxidised LDL-induced MCP-1 production by endothelial cells. Biochem Biophys Res Commun. 2004;318:680-3.

88. Springall $\mathrm{R}$, Amezcua-Guerra LM, Gonzalez-Pacheco $\mathrm{H}$, Furuzawa-Carballeda J, Gomez-Garcia L, Marquez-Velasco R, et al. Interferon-gamma increases the ratio of matrix metalloproteinase-9/tissue inhibitor of metalloproteinase- 1 in peripheral monocytes from patients with coronary artery disease. PLoS ONE. 2013;8:e72291.

89. Liang K, Dong SR, Peng H. Serum levels and clinical significance of IFN-gamma and IL-10 in patients with coronary heart disease. Eur Rev Med Pharmacol Sci. 2016;20:1339-43.

90. Yuan SM. alpha-Smooth muscle actin and ACTA2 gene expressions in vasculopathies. Braz J Cardiovasc Surg. 2015;30:644-9.

91. Logeswaran T, Friedburg $C$, Hofmann K, Akintuerk H, Biskup S, Graef $M$, et al. Two patients with the heterozygous $\mathrm{R} 189 \mathrm{H}$ mutation in ACTA2 and Complex congenital heart defects expands the cardiac phenotype of multisystemic smooth muscle dysfunction syndrome. Am J Med Genet A. 2017; 173:2566.

92. Schildmeyer LA, Braun R, Taffet G, Debiasi M, Burns AE, Bradley A, et al. Impaired vascular contractility and blood pressure homeostasis in the smooth muscle alpha-actin null mouse. FASEB J. 2000;14:2213-20.

93. Bennett MR, Sinha S, Owens GK. Vascular smooth muscle cells in atherosclerosis. Circ Res. 2016;118:692-702. 